Grace et al. | 1

\title{
Genomic identification, annotation, and comparative analysis of Vacuolar- type ATP synthase subunits in Diaphorina citri.
}

Rebecca Grace ${ }^{1,2}$ (ORCID: 0000-0003-4859-8331), Crissy Massimino ${ }^{1}$ (ORCID: 0000-00018097-7302), Teresa D. Shippy ${ }^{3}$ (ORCID: 0000-0003-2305-7432), Will Tank ${ }^{3}$ (ORCID: 00000002-2523-6090), Prashant S. Hosmani $^{4}$ (ORCID: 0000-0001-5722-4118), Mirella FloresGonzalez $^{4}$ (ORCID: 0000-0002-7759-1617), Lukas A. Mueller ${ }^{4}$ (ORCID: 0000-0001-86401750), Wayne B. Hunter ${ }^{5}$ (ORCID: 0000-0001-8603-3337), Joshua B. Benoit ${ }^{6}$ (ORCID: 00000002-4018-3513), Susan J. Brown ${ }^{3}$ (ORCID: 0000-0002-7984-0445), Tom D'Elia ${ }^{1}$ (ORCID: 0000-0003-2601-3128) and Surya Saha, ${ }^{4,7 *}($ ORCID: 0000-0002-1160-1413)

${ }^{1}$ Indian River State College, Fort Pierce, FL 34981, USA

${ }^{2}$ Department of Molecular Biology and Genetics, Cornell University, Ithaca, NY 14853, USA

${ }^{3}$ Division of Biology, Kansas State University, Manhattan, KS 66506, USA

${ }^{4}$ Boyce Thompson Institute, Ithaca, NY 14853, USA

${ }^{5}$ USDA-ARS, US Horticultural Research Laboratory, Fort Pierce, FL 34945, USA

${ }^{6}$ Department of Biological Sciences, University of Cincinnati, Cincinnati, OH 45221, USA

7 Animal and Comparative Biomedical Sciences, University of Arizona, Tucson, AZ 85721, USA

*suryasaha@cornell.edu 
Grace et al. $\mid 2$

\begin{abstract}
Detailed annotation and comparative analysis were performed on the Asian citrus psyllid (ACP), Diaphorina citri, vacuolar-type ATP synthase (V-ATPase) to support the biological understanding and development of novel therapeutics to manage psyllid vectors. $D$. citri is a hemipteran insect that vectors the causative agent, the bacteria Candidatus Liberibacter asiaticus (CLas), of the citrus greening disease, Huanglongbing (HLB). Millions of citrus trees have been destroyed by citrus greening and every grove in Florida has been directly impacted. In eukaryotic organisms, V-ATPase is an abundant heterodimeric enzyme that serves the cell with essential compartment acidification through the active processes that transport protons across the membrane. Manual curation was completed on 15 putative $V$-ATPase genes in the $D$. citri genome. Comparative genomic analysis reveals that the D. citri V-ATPase subunits share domains and motifs with other insects, including the V-ATPase-A superfamily domain from the V-ATPase catalytic subunit A, which shares a 92\% identity with Acyrthosiphon pisum. Phylogenetic analysis separates D. citri V-ATPase subunits into expected clades with orthologous sequences. Based on the results of annotation and comparative genomic analysis, RNAi therapies targeting D. citri V-ATPase genes, which have been successfully utilized in related hemipterans, are being pursued. Annotation of the D. citri genome is a critical step towards the development of directed-pest management that will lead to the reduced spread of the pathogens causing HLB throughout the citrus industry.
\end{abstract}

Research Area: Genetics and Genomics

Classifications: Animal Genetics, Bioinformatics 
Grace et al. | 3

\section{Data Description}

\section{Introduction}

Vacuolar $\left(\mathrm{H}^{+}\right)$-ATP synthase (V-ATPase) is a highly conserved eukaryotic enzyme [1]. Originally identified in the vacuole membrane, V-ATPase has a critical function in the plasma membrane and endomembrane system of almost every cell [2,3]. V-ATPase works to regulate the acidity of organelles, such as vacuoles, the Golgi apparatus, and coated vesicles, by translocating protons across their membranes and powering secondary transport processes. Structurally, V-ATPase has a noncatalytic transmembrane domain, the $\mathrm{V}_{0}$ rotor, and a catalytic cytoplasmic domain, the $\mathrm{V}_{1}$ stator. V-ATPase hydrolyzes adenosine triphosphate (ATP) into adenosine diphosphate (ADP), thus acting opposite of the related F-ATPase [1]. In insects, 13 protein subunits are typically required to build a single V-ATPase [4]. The $\mathrm{V}_{0}$ domain consists of subunits a through e and $\mathrm{V}_{1}$ consists of subunits $\mathrm{A}$ through $\mathrm{H}$ [5]. There is also a critical accessory subunit S1 (Ac45) that helps assemble the enzyme [6].

\section{Context}

In insects, high levels of V-ATPase are found in epithelial cells, and they are especially important in the digestive tract, helping to regulate nutrient uptake and solute transport [7]. Studies in several phyla, including insects, have demonstrated the lethality of silencing individual $V$-ATPase genes, making $V$-ATPase an attractive target for RNA interference (RNAi)based pest control $[1,4,7]$. We have characterized the genes encoding V-ATPase subunits in Diaphorina citri (Hemiptera: Liviidae; NCBI:txid121845) as a step towards the development of future management strategies to reduce the psyllid vector of the causative agent of 
Grace et al. 14

Huanglongbing (HLB), also known as citrus greening disease, the bacteria Candidatus Liberibacter asiaticus (CLas).

\section{Methods}

Vacuolar ATP synthase insect orthologs from Acyrthosiphon pisum (pea aphid) were obtained from the KEGG database (RRID:SCR_012773). Additional ortholog subunits occurring in noninsect eukaryotes, like Homo sapiens, were obtained from HUGO Gene Nomenclature Committee (HGNC) (RRID:SCR_002827) and the non-redundant NCBI Reference Sequence database [8]. V-ATPase protein sequences were used to query the predicted protein set from the D. citri MCOT (Maker (RRID:SCR_005309), Cufflinks (RRID:SCR_014597), Oases (RRID:SCR_011896), and Trinity (RRID:SCR_013048)) transcriptome via BLASTp [9].

Reciprocal BLASTp analysis was performed to validate the D. citri MCOT significant hits using the NCBI non-redundant protein database [8]. D. citri V-ATPase genes were identified in the genome (version 1.91) by searching for the identified mapped MCOT models in the WebApollo (RRID:SCR_005321) system hosted at Boyce Thompson Institute. Multiple alignments of the predicted D. citri MCOT proteins, other gene model sequences, and insect V-ATPase orthologs were performed using the European Bioinformatics Institute MUSCLE alignment online tool (RRID:SCR_004727) [10]. Further analysis using RNA-seq reads, Illumina DNA-seq reads, StringTie models, and PacBio Iso-seq transcripts were used to manually annotate the final $V$ ATPase gene models. Manually annotated $V$-ATPase gene models were then integrated into the version 3.0 Official Gene Set (OGS). V-ATPase genes were verified in WebApollo through analysis using de novo-assembled transcripts, Iso-seq transcripts, Augustus models, Mikado transcriptome, SwissProt proteins, and SNAP prediction models. A list of annotated D. citri 
Grace et al. $\mid 5$

identifiers and a sampling of evidence that supports the annotated models are found in Table 1. A more detailed description of the annotation workflow is available (Figure 1) [11]. V-ATPase nomenclature is somewhat inconsistent in the literature and between species, therefore, we have used nomenclature standards reported in previous work on other Hemiptera $[12,13]$.

Table 1. Evidence for gene annotations. MCOT transcriptome identifiers included, if applicable.

\begin{tabular}{|c|c|c|c|c|c|c|}
\hline Gene & Identifier & MCOT & $\begin{array}{c}\text { de novo } \\
\text { transcriptome }\end{array}$ & $\begin{array}{l}\text { Iso- } \\
\text { seq }\end{array}$ & $\begin{array}{l}\text { RNA- } \\
\text { seq }\end{array}$ & Ortholog \\
\hline$V$-ATPase al & Dcitr07g04330.1.1 & MCOT05340.0.CO & $\mathrm{X}$ & $X$ & $\mathrm{X}$ & $\mathrm{X}$ \\
\hline V-ATPase a2 & Dcitr07g01670.1.1 & MCOT20572.0.CT & $\mathrm{X}$ & $X$ & $X$ & $X$ \\
\hline$V$-ATPase $b$ & Dcitr12g08560.1.1 & - & $\mathrm{X}$ & $X$ & $X$ & $X$ \\
\hline$V$-ATPase c & Dcitr06g11110.1.1 & - & $\mathrm{X}$ & $\mathrm{X}$ & $\mathrm{X}$ & $\mathrm{X}$ \\
\hline V-ATPase d & Dcitr04g06930.1.1 & - & - & $X$ & $X$ & $X$ \\
\hline$V$-ATPase e & Dcitr03g19730.1.1 & - & $\mathrm{X}$ & $\mathrm{X}$ & $\mathrm{X}$ & $\mathrm{X}$ \\
\hline V-ATPase A & Dcitr06g09110.1.1 & MCOT04747.0.CC & - & $X$ & $\mathrm{X}$ & $\mathrm{X}$ \\
\hline V-ATPase B & Dcitr09g08730.1.1 & - & $\mathrm{X}$ & $\mathrm{X}$ & $\mathrm{X}$ & $\mathrm{X}$ \\
\hline$V$-ATPase C & Dcitr02g01535.1.1 & - & $X$ & $X$ & $\mathrm{X}$ & $\mathrm{X}$ \\
\hline V-ATPase D & Dcitr09g02030.1.1 & - & - & $X$ & $\mathrm{X}$ & $\mathrm{X}$ \\
\hline$V$-ATPase E & Dcitr04g09575.1.1 & - & - & $X$ & $X$ & $X$ \\
\hline$V$-ATPase F & Dcitr07g06920.1.1 & MCOT14638.0.CC & $X$ & $X$ & $X$ & $X$ \\
\hline$V$-ATPase G & Dcitr11g08810.1.1 & MCOT22289.0.CT & - & $\mathrm{X}$ & $\mathrm{X}$ & $X$ \\
\hline
\end{tabular}


Grace et al. |6

\author{
V-ATPase $H$ \\ (partial, N- Dcitr00g06320.1.1 \\ terminus)
}

V-ATPase H

(partial, C-

terminus)

Dcitr01g01240.1.1

MCOT00604.0.CT

- $\quad$ X $\quad X$

$\mathrm{X}$

$V$-ATPase

Ac45

$\mathrm{X}$

X X

$\mathrm{X}$

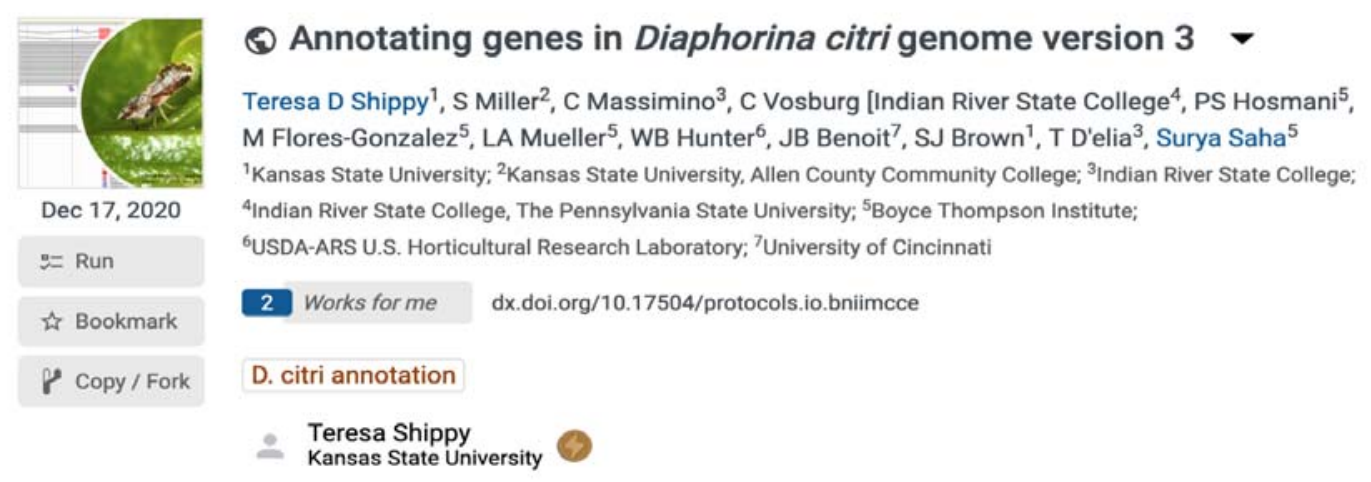

Figure 1. Protocols.io protocol for psyllid genome curation [11].

Reciprocal BLASTp of manually annotated v3.0 V-ATPase genes were performed at NCBI comparing the Insecta taxid. Insect orthologs from Acyrthosiphon pisum (pea aphid) [14,15], Bemisia tabaci (whitefly) [16], Aedes aegypti (yellow fever mosquito) [17], Apis mellifera (honeybee) [18], Tribolium castaneum (red flour beetle) [19], and Drosophila melanogaster (fruit fly) [20] were obtained by reciprocal BLASTp (RRID:SCR_004870) analysis of the nonredundant protein database at NCBI [8]. A neighbor-joining phylogenetic tree using the MUSCLE (RRID:SCR_011812) multiple sequence alignment with Poisson correction method and 1000 replicate bootstrap test was constructed using full-length protein sequences in MEGA version 7 (RRID:SCR_000667) for the transmembrane complex, the catalytic complex, and the accessory subunit Ac45, respectively (Figures 2-4) [21]. The sequence accession numbers used 
in these analyses can be found in Tables 2, 3, and 4. Comparative expression levels of D. citri $V$ ATPases throughout egg, nymph, and adult life stages in D. citri insects both exposed and not exposed to CLas were determined using RNA-seq data and the Citrus Greening Expression Network (CGEN) [9]. These gene expression levels were visualized using the pheatmap package in R (RRID:SCR_016418) [22,23]. Expression values for all samples discussed in this manuscript are visualized in Figures 5 and 6 and are reported as transcripts per million (TPM) in Table 5 . 


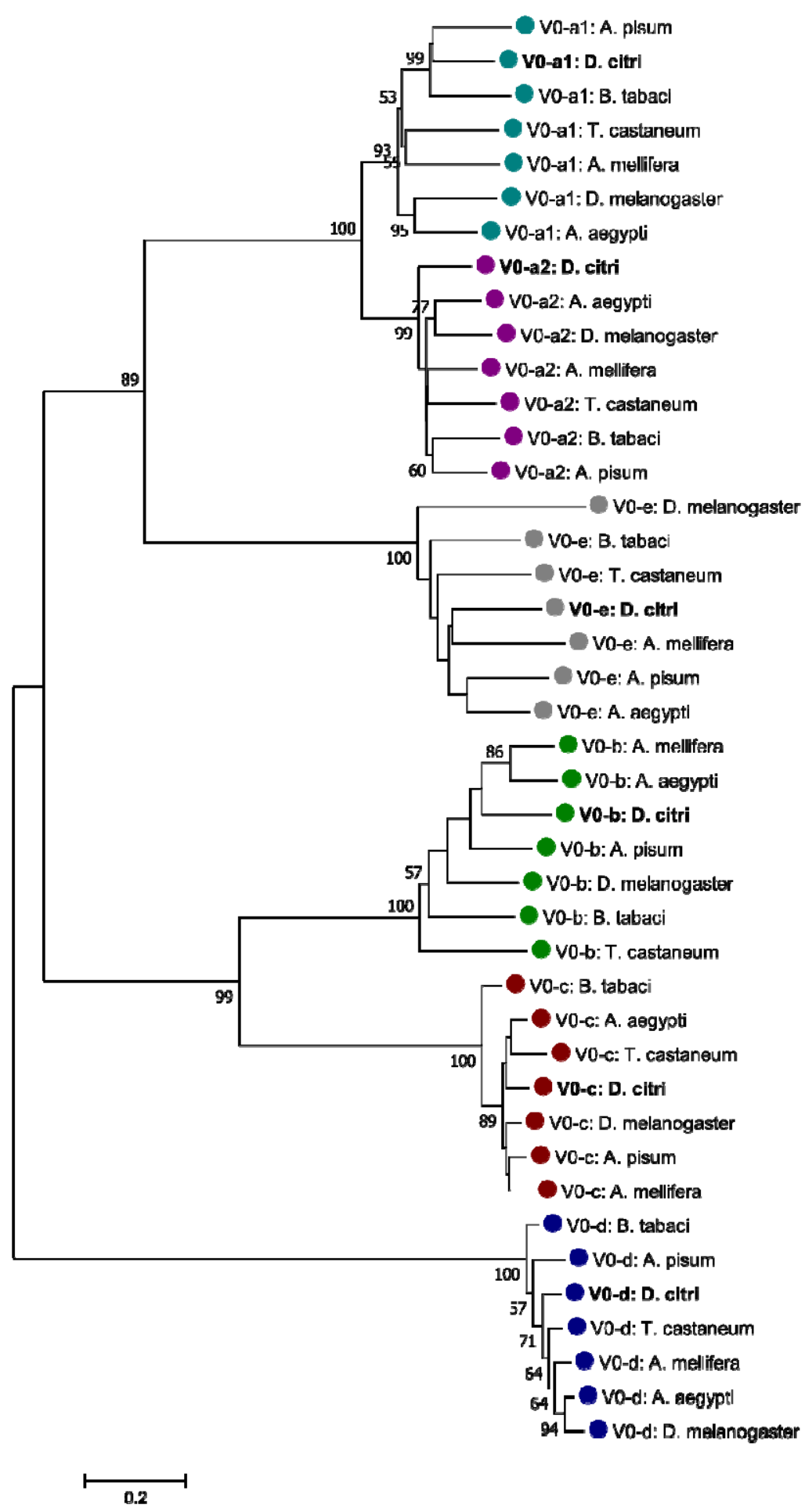

Figure 2. Phylogenetic analysis of V-ATPase $\mathrm{V}_{0}$, transmembrane domain, subunits a-e. The tree was constructed with MEGA7 software [21] using MUSCLE for alignment of amino acid sequences, followed by neighbor-joining analysis with 1000 bootstrap replications. Values 
bioRxiv preprint doi: https://doi.org/10.1101/2021.10.18.464890; this version posted October 19, 2021. The copyright holder for this preprint (which was not certified by peer review) is the author/funder, who has granted bioRxiv a license to display the preprint in perpetuity. It is made available under aCC-BY 4.0 International license.

Grace et al. $\mid 9$

greater than 50 are shown at nodes. D. citri is marked in bold and color-coding indicates specific $\mathrm{V}_{0}$ subunit groups. NCBI accession numbers are shown in Table 2. 
bioRxiv preprint doi: https://doi.org/10.1101/2021.10.18.464890; this version posted October 19,2021 . The copyright holder for this preprint (which was not certified by peer review) is the author/funder, who has granted bioRxiv a license to display the preprint in perpetuity. It is made available under aCC-BY 4.0 International license.

Grace et al. | 10

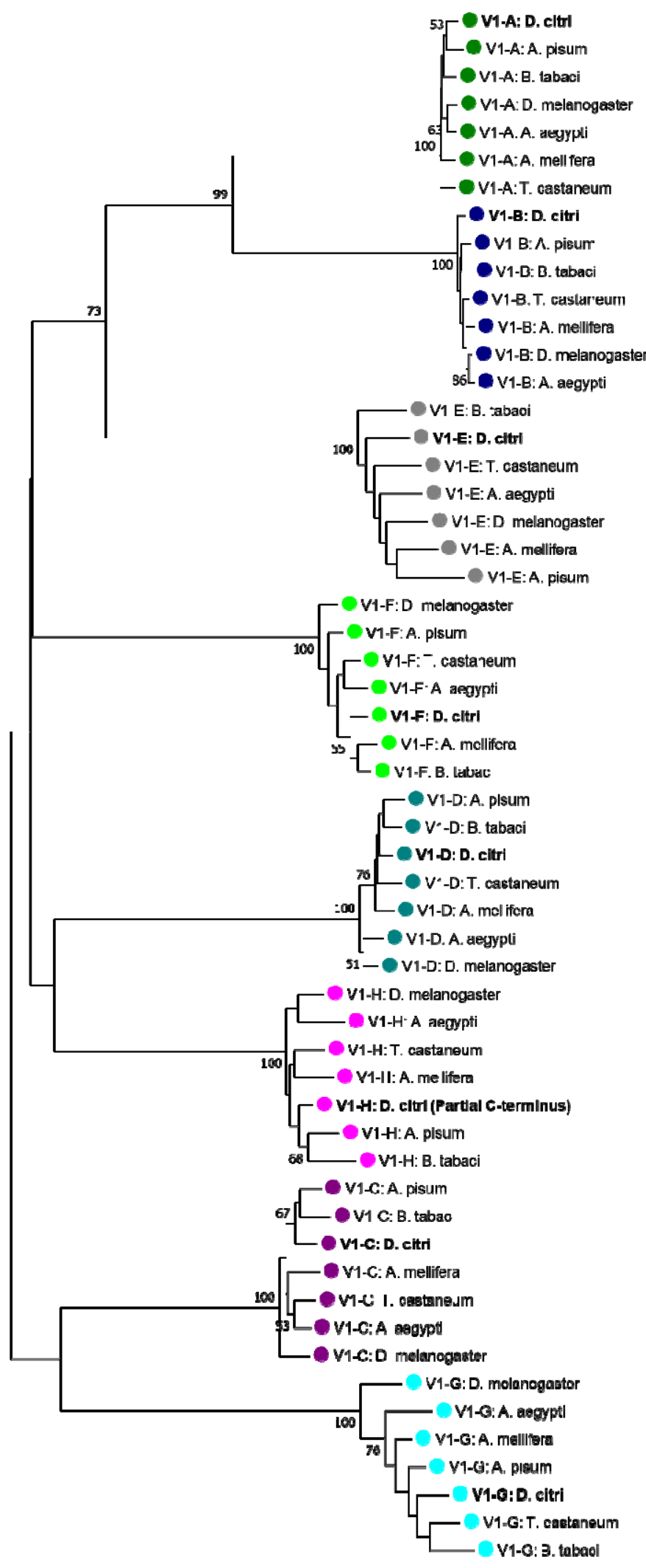


Figure 3. Phylogenetic analysis of V-ATPase $V_{1}$, catalytic domain, subunits A-G. The tree was constructed with MEGA7 [21] software using MUSCLE for alignment of amino acid sequences, followed by neighbor-joining analysis with 1000 bootstrap replications. Values greater than 50 are shown at nodes. D. citri is marked in bold and color-coding indicates specific $\mathrm{V}_{1}$ subunit groups. D. citri V-ATPase $H\left(\mathrm{~V}_{1}-\mathrm{H}\right)$ was annotated as two partial gene models, therefore, only the C-terminus, partial amino acid sequence of $\mathrm{V}_{1}-\mathrm{H}$ was included in this analysis. NCBI accession numbers are shown in Table 3.

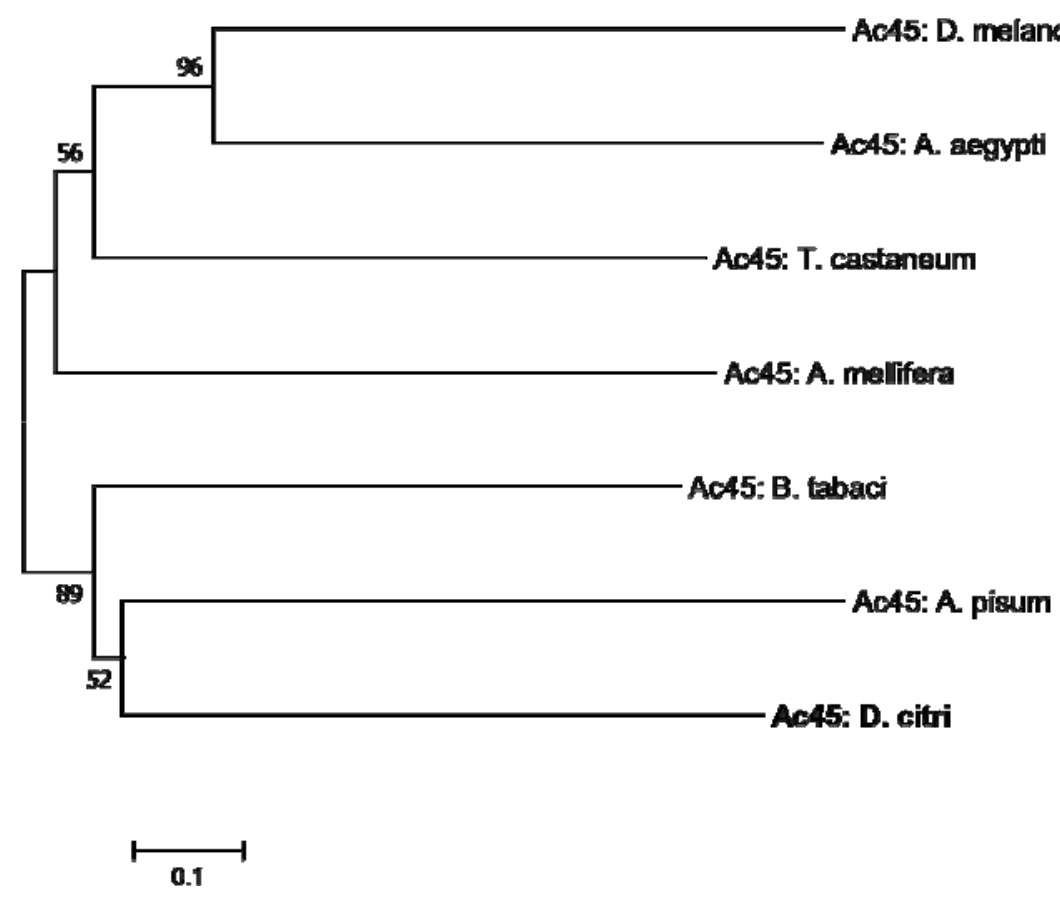

Figure 4. Phylogenetic analysis of V-ATPase accessory subunit Ac45. The tree was constructed with MEGA7 software [21] using MUSCLE for alignment of amino acid sequences, followed by neighbor-joining analysis with 1000 bootstrap replications. Values greater than 50 are shown at nodes. D. citri is marked in bold. NCBI accession numbers are shown in Table 4. 
Table 2. V-ATPase transmembrane subunit $\left(\mathrm{V}_{0}\right)$ BLAST table.

\begin{tabular}{|c|c|c|c|c|c|c|c|}
\hline Gene & & A. pisum & B. tabaci & T. castaneum & D. melanogaster & A. aegypti & A. mellifera \\
\hline \multirow{4}{*}{$\begin{array}{l}\text { V-ATPase } \\
\text { al }\end{array}$} & Accession & XP_029343960.1 & XP_018903507.1 & XP_008200952.1 & NP_650722.1 & XP_021706364.1 & XP_006565533.1 \\
\hline & Bit score & 1332 & 1312 & 1128 & 1151 & 1217 & 1166 \\
\hline & $\mathrm{QC}$ & $99 \%$ & $100 \%$ & $100 \%$ & $99 \%$ & $99 \%$ & $99 \%$ \\
\hline & Identity & $75.21 \%$ & $72.63 \%$ & $66.59 \%$ & $66.23 \%$ & $69.75 \%$ & $68.56 \%$ \\
\hline \multirow{4}{*}{$\begin{array}{l}\text { V-ATPase } \\
\text { a2 }\end{array}$} & Accession & XP_008183003.1 & XP_018913655.1 & XP_008200806.1 & NP_733274.1 & XP_021693139.1 & XP_026298707.1 \\
\hline & Bit score & 1369 & 1334 & 1331 & 1286 & 1369 & 1392 \\
\hline & QC & $100 \%$ & $100 \%$ & $100 \%$ & $100 \%$ & $100 \%$ & $100 \%$ \\
\hline & Identity & $79.10 \%$ & $76.42 \%$ & $76.18 \%$ & $76.19 \%$ & $77.21 \%$ & $79.05 \%$ \\
\hline \multirow{4}{*}{$V$-ATPase $b$} & Accession & NP_001155679.1 & XP_018909463.1 & NP_001161226.1 & NP_001247111.1 & XP_001662256.1 & XP_392599.1 \\
\hline & Bit score & 294 & 283 & 231 & 270 & 275 & $300^{-}$ \\
\hline & QC & $99 \%$ & $99 \%$ & $98 \%$ & $99 \%$ & $99 \%$ & $99 \%$ \\
\hline & Identity & $73.79 \%$ & $73.30 \%$ & $63.05 \%$ & $72.95 \%$ & $74.4 \%$ & $76.33 \%$ \\
\hline \multirow{4}{*}{ V-ATPase c } & Accession & NP_001155531.1 & XP_018897791.1 & XP_967959.1 & NP_476801.1 & XP_001654757.1 & NP_001011570.1 \\
\hline & Bit score & 268 & 250 & 258 & 271 & 267 & 259 \\
\hline & $\mathrm{QC}$ & $98 \%$ & $99 \%$ & $98 \%$ & $99 \%$ & $98 \%$ & $98 \%$ \\
\hline & Identity & $92.11 \%$ & $86.93 \%$ & $89.54 \%$ & $92.16 \%$ & $92.76 \%$ & $89.47 \%$ \\
\hline \multirow{4}{*}{ V-ATPase d } & Accession & NP_001191854.1 & XP_018903442.1 & XP_974905.1 & NP_570080.1 & XP_001661299.1 & XP_393438.2 \\
\hline & Bit score & 656 & 669 & 671 & 661 & 677 & 679 \\
\hline & $\mathrm{QC}$ & $99 \%$ & $100 \%$ & $100 \%$ & $99 \%$ & $100 \%$ & $100 \%$ \\
\hline & Identity & $89.60 \%$ & $91.95 \%$ & $91.67 \%$ & $90.20 \%$ & $91.95 \%$ & $92.82 \%$ \\
\hline \multirow{4}{*}{ V-ATPase e } & Accession & XP_003242132.1 & XP_018909271.1 & XP_971898.1 & NP_001097499.1 & ABF18129.1 & XP_624787.1 \\
\hline & Bit score & $127^{-}$ & 119 & 123 & $106^{-}$ & 133 & $127^{-}$ \\
\hline & $\mathrm{QC}$ & $96 \%$ & $96 \%$ & $94 \%$ & $96 \%$ & $100 \%$ & $94 \%$ \\
\hline & Identity & $69.51 \%$ & $70.73 \%$ & $72.50 \%$ & $58.54 \%$ & $70.59 \%$ & $71.25 \%$ \\
\hline
\end{tabular}

Accession number, bit score, query coverage (QC), and identity results from protein BLAST analysis of annotated D. citri V-ATPase transmembrane subunit genes

to

their

putative

orthologs. 
Table 3. V-ATPase catalytic subunit $\left(\mathrm{V}_{1}\right)$ BLAST table.

\begin{tabular}{|c|c|c|c|c|c|c|c|}
\hline Gene & & A. pisum & B. tabaci & T. castaneum & D. melanogaster & A. aegypti & A. mellifera \\
\hline \multirow{4}{*}{ V-ATPase A } & Accession & XP_008179407.1 & XP_018897790.1 & XP_976188.1 & NP_001246015.1 & XP_021709029.1 & XP_016769524.1 \\
\hline & Bit score & 1176 & 1187 & 1167 & 1162 & 1168 & 1174 \\
\hline & $\mathrm{QC}$ & $99 \%$ & $99 \%$ & $99 \%$ & $99 \%$ & $100 \%$ & $99 \%$ \\
\hline & Identity & $91.84 \%$ & $92.47 \%$ & $90.21 \%$ & $90.18 \%$ & $90.57 \%$ & $90.72 \%$ \\
\hline \multirow{4}{*}{ V-ATPase B } & Accession & XP_003246082.1 & XP_018896879.1 & XP_967844.1 & NP_001163597.1 & XP_001651458.1 & XP_624112.1 \\
\hline & Bit score & 966 & 976 & 966 & 966 & 968 & 955 \\
\hline & $\mathrm{QC}$ & $98 \%$ & $99 \%$ & $98 \%$ & $98 \%$ & $98 \%$ & $98 \%$ \\
\hline & Identity & $93.88 \%$ & $94.33 \%$ & $93.48 \%$ & $93.88 \%$ & $94.09 \%$ & $92.87 \%$ \\
\hline \multirow{4}{*}{ V-ATPase C } & Accession & XP_001946227.1 & XP_018915661.1 & XP_008195426.1 & NP_477266.1 & XP_021695404.1 & XP_006562159.1 \\
\hline & Bit score & 702 & 653 & 671 & 645 & 671 & 648 \\
\hline & $\mathrm{QC}$ & $99 \%$ & $99 \%$ & $98 \%$ & $98 \%$ & $99 \%$ & $99 \%$ \\
\hline & Identity & $87.05 \%$ & $82.81 \%$ & $82.20 \%$ & $79.11 \%$ & $81.77 \%$ & $79.95 \%$ \\
\hline \multirow{4}{*}{ V-ATPase D } & Accession & NP_001119691.1 & XP_018904914.1 & XP_975872.1 & NP_651987.1 & XP_001660426.1 & XP_394769.2 \\
\hline & Bit score & 420 & 423 & 426 & 408 & 401 & 404 \\
\hline & $\mathrm{QC}$ & $100 \%$ & $100 \%$ & $100 \%$ & $100 \%$ & $100 \%$ & $100 \%$ \\
\hline & Identity & $87.24 \%$ & $86.42 \%$ & $86.53 \%$ & $82.52 \%$ & $80.89 \%$ & $86.94 \%$ \\
\hline \multirow{4}{*}{ V-ATPase E } & Accession & NP_001155650.1 & XP_018901912.1 & XP_970621.1 & NP_001287182.1 & XP_001655825.1 & XP_625098.1 \\
\hline & Bit score & 301 & 336 & 341 & 325 & 335 & 336 \\
\hline & QC & $99 \%$ & $100 \%$ & $100 \%$ & $100 \%$ & $100 \%$ & $100 \%$ \\
\hline & Identity & $73.21 \%$ & $71.68 \%$ & $73.01 \%$ & $71.24 \%$ & $70.80 \%$ & $73.01 \%$ \\
\hline \multirow{4}{*}{ V-ATPase F } & Accession & NP_001119690.1 & XP_018905603.1 & XP_975016.1 & NP_476969.1 & XP_001655376.1 & XP_624852.1 \\
\hline & Bit score & 223 & 227 & 216 & 217 & 216 & 230 \\
\hline & QC & $98 \%$ & $98 \%$ & $98 \%$ & $98 \%$ & $100 \%$ & $98 \%$ \\
\hline & Identity & $85.25 \%$ & $88.52 \%$ & $82.79 \%$ & $81.15 \%$ & $82.26 \%$ & $88.52 \%$ \\
\hline \multirow{2}{*}{$V$-ATPase G } & Accession & NP_001119628.1 & XP_018908074.1 & XP_973974.1 & NP_001287407.1 & XP_001652605.1 & XP_624346.1 \\
\hline & Bit score & 184 & 188 & 173 & 154 & 171 & 180 \\
\hline
\end{tabular}


Grace et al. $\mid 14$

\begin{tabular}{|c|c|c|c|c|c|c|c|}
\hline & QC & $99 \%$ & $100 \%$ & $98 \%$ & $96 \%$ & $96 \%$ & $98 \%$ \\
\hline & Identity & $80.34 \%$ & $81.51 \%$ & $74.14 \%$ & $65.79 \%$ & $73.68 \%$ & $76.72 \%$ \\
\hline \multirow{4}{*}{$\begin{array}{l}V \text {-ATPase H } \\
\text { (partial, N- } \\
\text { terminus) }\end{array}$} & Accession & XP_001949116.3 & XP_018904009.1 & NP_001280516.1 & NP_001260510.1 & XP_001652018.1 & XP_003251675.1 \\
\hline & Bit score & 132 & 162 & 132 & 110 & 130 & 142 \\
\hline & QC & $90 \%$ & $99 \%$ & $99 \%$ & $84 \%$ & $91 \%$ & $94 \%$ \\
\hline & Identity & $57.69 \%$ & $68.42 \%$ & $57.26 \%$ & $56.70 \%$ & $58.10 \%$ & $64.22 \%$ \\
\hline \multirow{4}{*}{$\begin{array}{l}V \text {-ATPase H } \\
\text { (partial, C- } \\
\text { terminus) }\end{array}$} & Accession & XP_001949116.3 & XP_018904009.1 & NP_001280516.1 & NP_001260510.1 & XP_001652018.1 & XP_003251675.1 \\
\hline & Bit score & 530 & 506 & 513 & 493 & 498 & 490 \\
\hline & QC & $100 \%$ & $99 \%$ & $100 \%$ & $100 \%$ & $100 \%$ & $99 \%$ \\
\hline & Identity & $83.95 \%$ & $82.33 \%$ & $83.28 \%$ & $77.59 \%$ & $78.93 \%$ & $82.15 \%$ \\
\hline
\end{tabular}

Accession number, bit score, query coverage (QC), and identity results from protein BLAST analysis of annotated D. citri V-ATPase catalytic subunit genes to their putative orthologs.

Table 4. V-ATPase Ac45, accessory subunit, BLAST table.

\begin{tabular}{llllllll}
\hline \multirow{2}{*}{ Gene } & \multicolumn{2}{c}{ A. pisum } & B.tabaci & T. castaneum & D. melanogaster & A. aegypti & A. mellifera \\
& & & & & & \\
\hline \multirow{4}{*}{ Ac45 } & Accession & NP_001162140.1 & XP_018899028.1 & XP_974187.2 & NP_610470.1 & XP_001658652.1 XP_001121483.3 \\
& Bit score & 136 & 177 & 114 & 77.4 & 92.4 & 88.6 \\
& QC & $83 \%$ & $97 \%$ & $100 \%$ & $96 \%$ & $95 \%$ & $93 \%$ \\
& Identity & $30.87 \%$ & $33.49 \%$ & $27.03 \%$ & $24.94 \%$ & $23.81 \%$ & $26.35 \%$ \\
\hline
\end{tabular}

Accession number, bit score, query coverage (QC), and identity results from protein BLAST analysis of annotated D. citri V-ATPase
Ac45
gene
to
its
putative
orthologs. 
bioRxiv preprint doi: https://doi.org/10.1101/2021.10.18.464890; this version posted October 19,2021 . The copyright holder for this preprint (which was not certified by peer review) is the author/funder, who has granted bioRxiv a license to display the preprint in perpetuity. It is made available under aCC-BY 4.0 International license.

Grace et al. $\mid 15$

(a)
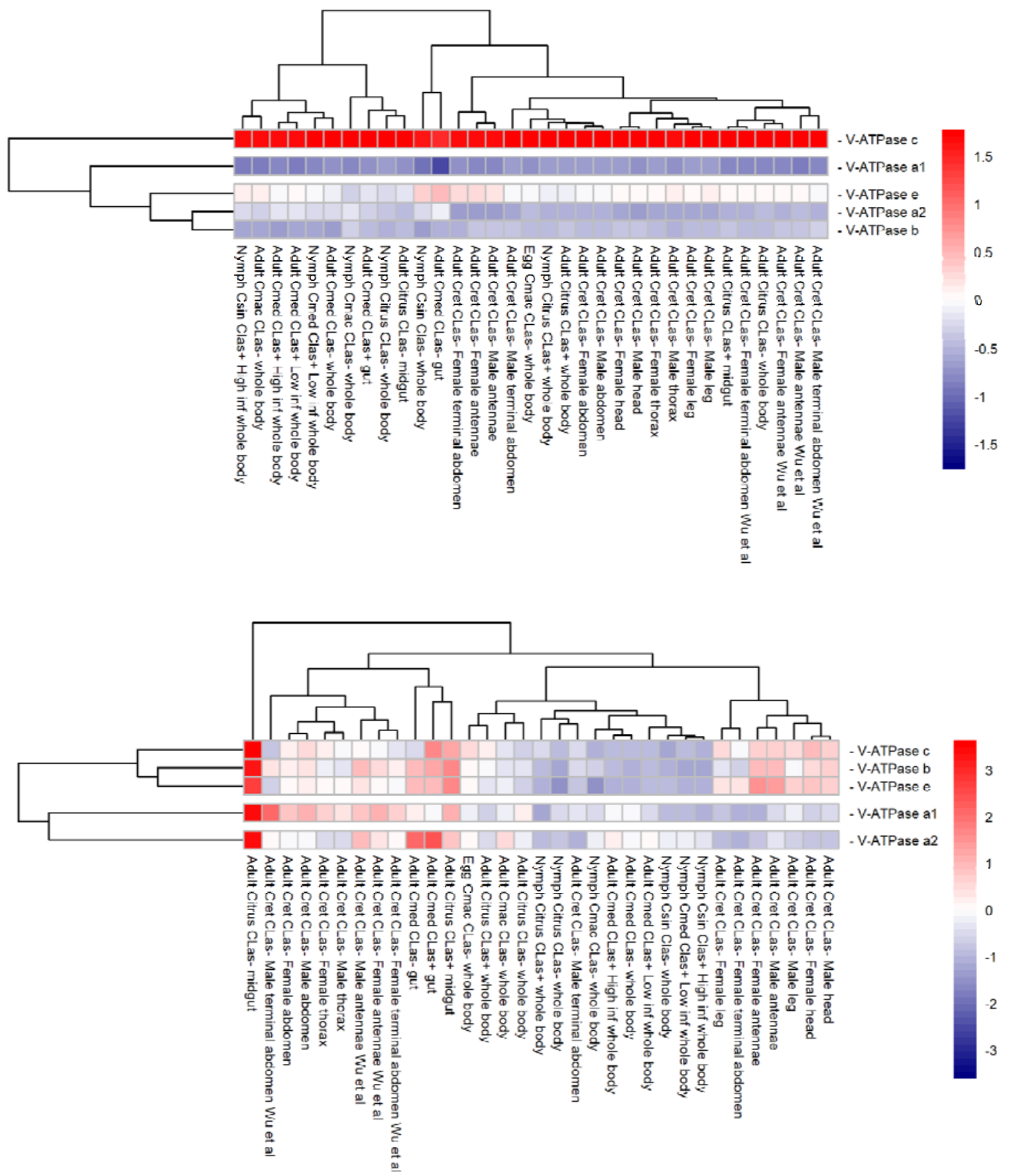

(b)

Figure 5. Comparative expression levels of the D. citri V-ATPase genes encoding the $\mathrm{V}_{0}$, transmembrane, subunits in $D$. citri insects reared on various infected and uninfected citrus varieties. Expression data were collected from the Citrus Greening Expression Network [9], with psyllid RNA-Seq data from NCBI BioProject's PRJNA609978 and PRJNA448935, in addition 
bioRxiv preprint doi: https://doi.org/10.1101/2021.10.18.464890; this version posted October 19,2021 . The copyright holder for this preprint (which was not certified by peer review) is the author/funder, who has granted bioRxiv a license to display the preprint in perpetuity. It is made available under aCC-BY 4.0 International license.

Grace et al. | 16

to several published datasets [24-28]. Citrus hosts are abbreviated as Csin (Citrus sinensis), Cmed (Citrus medica), Cret (Citrus reticulata), and Cmac (Citrus macrophylla). TPM values are listed in Table 5. Rows of genes and columns of RNA-Seq data are clustered based on expression differences. (a) Expression data scaled by sample. (b) Expression data scaled by gene.

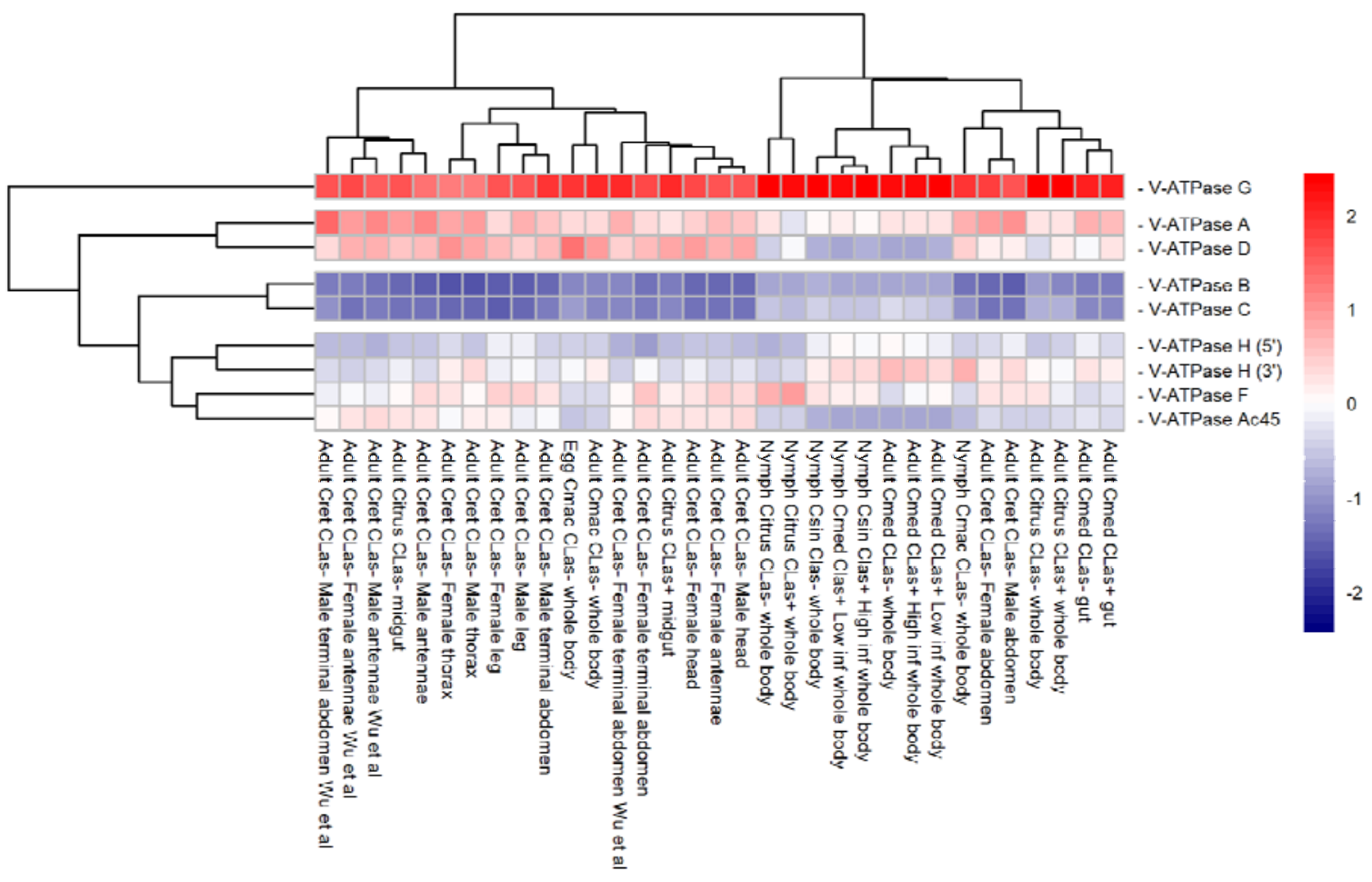

(a) 
Grace et al. | 17

(b)

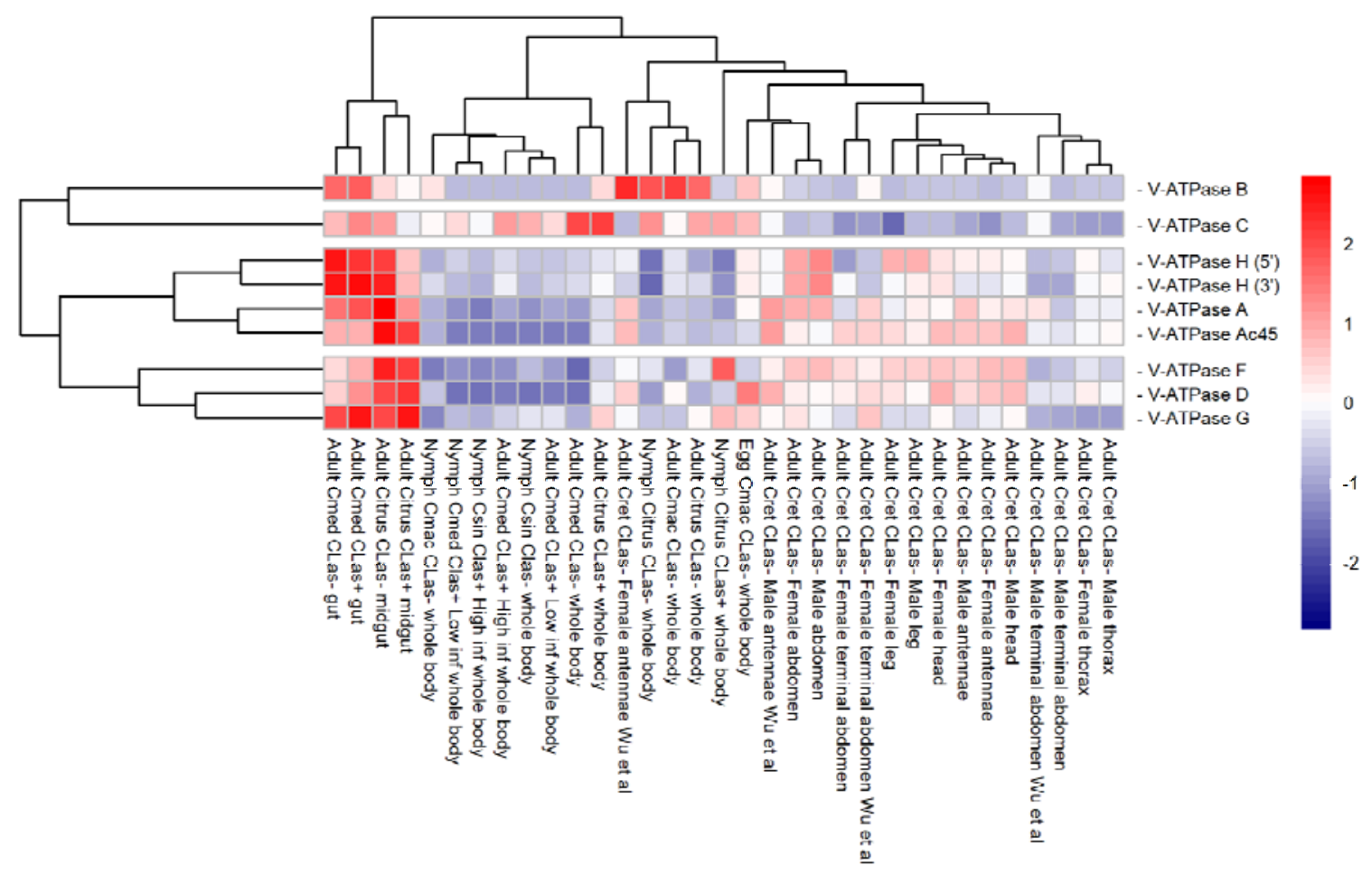

Figure 6. Comparative expression levels of the D. citri V-ATPase genes encoding the $\mathrm{V}_{1}$, catalytic, and accessory subunits in $D$. citri insects reared on various infected and uninfected citrus varieties. V-ATPase $H$ was annotated as two partial models and are both represented here separately as V-ATPase $H\left(5^{\prime}\right)$, denoting the 5-prime end of the gene, and $V$-ATPase $H\left(3^{\prime}\right)$, denoting the 3-prime end of the gene. Expression data were collected from the Citrus Greening Expression Network [9], with psyllid RNA-Seq data from NCBI BioProject's PRJNA609978 and PRJNA448935, in addition to several published datasets [24-28]. Citrus hosts are abbreviated as Csin (Citrus sinensis), Cmed (Citrus medica), Cret (Citrus reticulata), and Cmac (Citrus macrophylla). TPM values are listed in Table 5. Rows of genes and columns of RNASeq data are clustered based on expression differences. (a) Expression data scaled by sample. (b) Expression data scaled by gene. 
Table 5. Expression values listed as TPM, visualized in Figures 5 and 6.

\begin{tabular}{|c|c|c|c|c|c|c|c|c|c|c|c|c|c|c|}
\hline Gene name & $\begin{array}{c}V- \\
\text { ATPase } \\
\text { al }\end{array}$ & $\begin{array}{c}V- \\
\text { ATPase } \\
a 2\end{array}$ & $\begin{array}{c}V- \\
\text { ATPase } \\
b\end{array}$ & $\begin{array}{c}V- \\
\text { ATPase } \\
c\end{array}$ & $\begin{array}{c}V- \\
\text { ATPase } \\
e\end{array}$ & $\begin{array}{c}V- \\
\text { ATPase } \\
A\end{array}$ & $\begin{array}{c}V- \\
\text { ATPase } \\
B\end{array}$ & $\begin{array}{c}V- \\
\text { ATPase } \\
\text { C }\end{array}$ & $\begin{array}{c}V- \\
\text { ATPase } \\
D\end{array}$ & $\begin{array}{c}V- \\
\text { ATPase } \\
F\end{array}$ & $\begin{array}{c}V- \\
\text { ATPase } \\
G\end{array}$ & $\begin{array}{c}V- \\
\text { ATPase } \\
H\left(5^{\prime}\right)\end{array}$ & $\begin{array}{c}V- \\
\text { ATPase } \\
H\left(3^{\prime}\right)\end{array}$ & $\begin{array}{c}V \text { - } \\
\text { ATPase } \\
\text { Ac45 }\end{array}$ \\
\hline Gene ID & $\begin{array}{c}\text { Dcitr07 } \\
\text { g04330 } \\
.1 .1\end{array}$ & $\begin{array}{c}\text { Dcitr07 } \\
\text { g01670 } \\
.1 .1\end{array}$ & $\begin{array}{c}\text { Dcitr12 } \\
\text { g08560 } \\
.1 .1\end{array}$ & $\begin{array}{c}\text { Dcitr06 } \\
\text { g11110 } \\
.1 .1\end{array}$ & $\begin{array}{c}\text { Dcitr03 } \\
\text { g19730 } \\
.1 .1\end{array}$ & $\begin{array}{c}\text { Dcitr06 } \\
\text { g09110 } \\
.1 .1\end{array}$ & $\begin{array}{c}\text { Dcitr09 } \\
\text { g08730 } \\
.1 .1\end{array}$ & $\begin{array}{c}\text { Dcitr02 } \\
\text { g01530 } \\
.1 .1\end{array}$ & $\begin{array}{c}\text { Dcitr09 } \\
\text { g02030 } \\
.1 .1\end{array}$ & $\begin{array}{c}\text { Dcitr07 } \\
\text { g06920 } \\
.1 .1\end{array}$ & $\begin{array}{c}\text { Dcitr11 } \\
\text { g08810 } \\
.1 .1\end{array}$ & $\begin{array}{c}\text { Dcitr00 } \\
\text { g06320 } \\
.1 .1\end{array}$ & $\begin{array}{c}\text { Dcitr01 } \\
\text { g01230 } \\
.1 .1\end{array}$ & $\begin{array}{c}\text { Dcitr09 } \\
\text { g09620 } \\
.1 .1\end{array}$ \\
\hline $\begin{array}{l}\text { Egg Cmac } \\
\text { CLas- whole } \\
\text { body }\end{array}$ & 27.02 & 128.27 & 134.58 & $\begin{array}{c}1085.0 \\
7\end{array}$ & 312.06 & 343.23 & 15.59 & 49.46 & 567.45 & 199.16 & 698.5 & 161.78 & 248.69 & 148.17 \\
\hline $\begin{array}{l}\text { Nymph Cmed } \\
\text { Clas+ Low } \\
\text { inf whole } \\
\text { body }\end{array}$ & 12.02 & 96.32 & 40.57 & 398.66 & 133.64 & 126.32 & 0 & 43.02 & 1.12 & 124.84 & 416.8 & 119.07 & 160.75 & 0 \\
\hline $\begin{array}{l}\text { Nymph Csin } \\
\text { Clas+ High } \\
\text { inf whole } \\
\text { body }\end{array}$ & 8.82 & 89.21 & 39.09 & 334.65 & 131.69 & 102.13 & 0 & 36.49 & 1.4 & 117.74 & 408.72 & 99.61 & 144.58 & 0 \\
\hline $\begin{array}{l}\text { Nymph Csin } \\
\text { Clas- whole } \\
\text { body }\end{array}$ & 12.99 & 99.94 & 49.5 & 312.43 & 161.29 & 134.19 & 0 & 51.94 & 2.57 & 176.32 & 539.91 & 104.1 & 155.61 & 0 \\
\hline $\begin{array}{l}\text { Nymph Cmac } \\
\text { CLas- whole } \\
\text { body }\end{array}$ & 21.77 & 98.95 & 82.02 & 340.27 & 83.11 & 189.27 & 11.78 & 37.44 & 164.67 & 97.37 & 293.69 & 91 & 191.48 & 69.04 \\
\hline $\begin{array}{l}\text { Nymph Citrus } \\
\text { CLas- whole } \\
\text { body }\end{array}$ & 20.57 & 58.34 & 42.83 & 399.58 & 83.86 & 168.7 & 28.72 & 58.81 & 80.9 & 223.67 & 439.82 & 39.64 & 73.2 & 81.35 \\
\hline $\begin{array}{l}\text { Nymph Citrus } \\
\text { CLas+ whole } \\
\text { body }\end{array}$ & 4.94 & 38.29 & 59.33 & 621.98 & 146.78 & 160.92 & 2.44 & 53.38 & 190.77 & 444.42 & 766.12 & 44.97 & 115.28 & 114.6 \\
\hline
\end{tabular}


Grace et al. | 19

\begin{tabular}{|c|c|c|c|c|c|c|c|c|c|c|c|c|c|c|}
\hline $\begin{array}{l}\text { Adult Cmed } \\
\text { CLas- gut }\end{array}$ & 38.18 & 289.58 & 200.59 & 647.9 & 426.18 & 605.11 & 26.07 & 50.69 & 381.68 & 296.91 & $\begin{array}{c}1044.0 \\
9\end{array}$ & 341.38 & 473.57 & 332.39 \\
\hline $\begin{array}{l}\text { Adult Cmed } \\
\text { CLas+ gut }\end{array}$ & 30.2 & 319.44 & 230.85 & $\begin{array}{c}1705.2 \\
5\end{array}$ & 428.81 & 659.96 & 27.78 & 60.44 & 529.68 & 352.93 & $\begin{array}{c}1163.0 \\
3\end{array}$ & 317.72 & 485.17 & 329.78 \\
\hline $\begin{array}{l}\text { Adult Cmed } \\
\text { CLas+ High } \\
\text { inf whole } \\
\text { body }\end{array}$ & 30.47 & 131.75 & 59.8 & 406.4 & 151.3 & 171.64 & 0 & 55.4 & 3.76 & 118.88 & 477.59 & 125.92 & 210.48 & 0 \\
\hline $\begin{array}{l}\text { Adult Cmed } \\
\text { CLas+ Low } \\
\text { inf whole } \\
\text { body }\end{array}$ & 20.42 & 129.81 & 62.36 & 433.76 & 165.49 & 170.73 & 0 & 43.57 & 3.03 & 137.14 & 519.39 & 105.76 & 181.58 & 0 \\
\hline $\begin{array}{l}\text { Adult Cmed } \\
\text { CLas- whole } \\
\text { body }\end{array}$ & 30.31 & 113.42 & 49.08 & 412.59 & 132.76 & 149.36 & 0 & 71.67 & 2.27 & 70.33 & 411.72 & 118.34 & 191.46 & 0 \\
\hline $\begin{array}{l}\text { Adult Cmac } \\
\text { CLas- whole } \\
\text { body }\end{array}$ & 26.08 & 162.08 & 102.83 & 676.39 & 273.28 & 230.43 & 31.71 & 38.6 & 306.37 & 142.53 & 456.71 & 128.13 & 200.35 & 122.02 \\
\hline $\begin{array}{l}\text { Adult Citrus } \\
\text { CLas- whole } \\
\text { body }\end{array}$ & 36.98 & 101.48 & 106.15 & 554.39 & 212.37 & 217.42 & 27.13 & 54.78 & 123.29 & 228.76 & 592.56 & 83.13 & 188.23 & 96.36 \\
\hline $\begin{array}{l}\text { Adult Citrus } \\
\text { CLas+ whole } \\
\text { body }\end{array}$ & 19.8 & 77.29 & 133.73 & 951.84 & 248.6 & 268.61 & 12.34 & 73.2 & 257.5 & 196.34 & 698.74 & 131.62 & 218.34 & 158.58 \\
\hline $\begin{array}{l}\text { Adult Citrus } \\
\text { CLas- midgut }\end{array}$ & 103.17 & 399.83 & 371.99 & $\begin{array}{c}2604.5 \\
9\end{array}$ & 688.74 & 825.5 & 13.38 & 56.53 & 681.66 & 519.97 & $\begin{array}{c}1032.3 \\
3\end{array}$ & 309.77 & 460.52 & 594.4 \\
\hline $\begin{array}{l}\text { Adult Citrus } \\
\text { CLas+ } \\
\text { midgut }\end{array}$ & 55.24 & 188.63 & 261.09 & $\begin{array}{c}1449.9 \\
5\end{array}$ & 529.49 & 549.52 & 8.89 & 33.98 & 732.48 & 484.27 & $\begin{array}{c}1170.8 \\
1\end{array}$ & 195.92 & 304.17 & 521.08 \\
\hline
\end{tabular}


Grace et al. $\mid 20$

\begin{tabular}{|c|c|c|c|c|c|c|c|c|c|c|c|c|c|c|}
\hline $\begin{array}{l}\text { Adult Cret } \\
\text { CLas- Female } \\
\text { abdomen }\end{array}$ & 48.78 & 117.05 & 150.69 & 1014.5 & 302.79 & 479.37 & 2.55 & 23.5 & 322.17 & 327.16 & 646.63 & 220.46 & 324.63 & 224.43 \\
\hline $\begin{array}{l}\text { Adult Cret } \\
\text { CLas- Female } \\
\text { antennae }\end{array}$ & 9.84 & 59.74 & 202.33 & $\begin{array}{c}1200.5 \\
8\end{array}$ & 515.42 & 374.86 & 0.79 & 16.81 & 407.15 & 343.42 & 564.73 & 165.93 & 204.21 & 300.71 \\
\hline $\begin{array}{l}\text { Adult Cret } \\
\text { CLas- Female } \\
\text { head }\end{array}$ & 19.05 & 57.69 & 174.15 & $\begin{array}{c}1299.6 \\
4\end{array}$ & 416.69 & 357.16 & 1.65 & 23.05 & 452.84 & 326.87 & 606.78 & 173.26 & 258.3 & 321.53 \\
\hline $\begin{array}{l}\text { Adult Cret } \\
\text { CLas- Female } \\
\text { leg }\end{array}$ & 13.96 & 40.8 & 110.91 & $\begin{array}{c}1106.2 \\
1\end{array}$ & 357.39 & 299.85 & 0.39 & 7.36 & 339.68 & 315.91 & 501.89 & 214.39 & 217.88 & 269.99 \\
\hline $\begin{array}{l}\text { Adult Cret } \\
\text { CLas- Female } \\
\text { terminal } \\
\text { abdomen }\end{array}$ & 10.27 & 28.28 & 80.92 & 857.87 & 355.37 & 274.98 & 0.46 & 15.78 & 334.95 & 305.78 & 513.38 & 72.89 & 219.72 & 273.18 \\
\hline $\begin{array}{l}\text { Adult Cret } \\
\text { CLas- Female } \\
\text { thorax }\end{array}$ & 45.76 & 77.36 & 118.42 & 971.53 & 318.3 & 310.44 & 1.83 & 18.82 & 327.81 & 228.02 & 352.69 & 154.87 & 226.17 & 201.78 \\
\hline $\begin{array}{l}\text { Adult Cret } \\
\text { CLas- Male } \\
\text { abdomen }\end{array}$ & 55.04 & 113.66 & 163.22 & $\begin{array}{c}1121.0 \\
3\end{array}$ & 329.87 & 476.84 & 2.13 & 25.6 & 308.86 & 333.54 & 580.91 & 251.62 & 353.14 & 203.71 \\
\hline $\begin{array}{l}\text { Adult Cret } \\
\text { CLas- Male } \\
\text { antennae }\end{array}$ & 21.34 & 88.88 & 202.61 & $\begin{array}{c}1216.6 \\
3\end{array}$ & 502.11 & 454.87 & 2.06 & 20.5 & 366.31 & 317.82 & 489.63 & 163.36 & 206.39 & 300.97 \\
\hline $\begin{array}{l}\text { Adult Cret } \\
\text { CLas- Male } \\
\text { head }\end{array}$ & 22.42 & 57.66 & 180.09 & $\begin{array}{c}1215.4 \\
9\end{array}$ & 397.1 & 382.37 & 1.84 & 22.9 & 427.39 & 340.13 & 591.69 & 156.61 & 223.47 & 333.35 \\
\hline $\begin{array}{l}\text { Adult Cret } \\
\text { CLas- Male } \\
\text { leg }\end{array}$ & 26.24 & 70.25 & 126.32 & $\begin{array}{c}1144.4 \\
3\end{array}$ & 386.63 & 368.02 & 2.26 & 24.38 & 312.31 & 317.42 & 503.46 & 216.72 & 245.72 & 225.41 \\
\hline
\end{tabular}


Grace et al. $\mid 21$

\begin{tabular}{|c|c|c|c|c|c|c|c|c|c|c|c|c|c|c|}
\hline $\begin{array}{l}\text { Adult Cret } \\
\text { CLas- Male } \\
\text { terminal } \\
\text { abdomen }\end{array}$ & 23.66 & 34.11 & 94.32 & 636.58 & 176.52 & 226.85 & 0.57 & 19.13 & 234.07 & 190.22 & 375.51 & 109.22 & 137.98 & 154.37 \\
\hline $\begin{array}{l}\text { Adult Cret } \\
\text { CLas- Male } \\
\text { thorax }\end{array}$ & 38.48 & 81.1 & 106.97 & 826.51 & 307.15 & 309.28 & 1.82 & 17.83 & 289.69 & 205.61 & 340.62 & 133.14 & 229.48 & 217.37 \\
\hline $\begin{array}{l}\text { Adult Cret } \\
\text { CLas- Female } \\
\text { antennae Wu } \\
\text { et al }\end{array}$ & 55.37 & 150.12 & 175.28 & 828.29 & 314.86 & 436.78 & 33.85 & 23.48 & 389.56 & 253.26 & 576.96 & 141.07 & 182.16 & 314.72 \\
\hline $\begin{array}{l}\text { Adult Cret } \\
\text { CLas- Female } \\
\text { terminal } \\
\text { abdomen } \mathrm{Wu} \\
\text { et al }\end{array}$ & 46.1 & 121.03 & 149.2 & 727.77 & 282.02 & 427.76 & 9.27 & 18.35 & 373.06 & 280.05 & 731.99 & 106.17 & 164.41 & 273.56 \\
\hline $\begin{array}{l}\text { Adult Cret } \\
\text { CLas- Male } \\
\text { antennae Wu } \\
\text { et al }\end{array}$ & 48.09 & 190.97 & 202.67 & 923.05 & 349.45 & 524.4 & 8.74 & 35.65 & 448.15 & 268.8 & 602.45 & 147.99 & 218.22 & 369.43 \\
\hline $\begin{array}{l}\text { Adult Cret } \\
\text { CLas- Male } \\
\text { terminal } \\
\text { abdomen } \mathrm{Wu} \\
\text { et al }\end{array}$ & 77.21 & 129.36 & 162.97 & 503.06 & 208.23 & 381.74 & 8.29 & 36.86 & 235.06 & 163.07 & 405.46 & 95.28 & 131.34 & 183.8 \\
\hline
\end{tabular}

Comparative expression levels in transcripts per million (TPM) of the D. citri V-ATPase genes encoding the $\mathrm{V}_{\text {-ATPase }} \mathrm{V}_{0}$ transmembrane, $\mathrm{V}_{1}$ catalytic, and accessory subunits in $D$. citri insects reared on various infected and uninfected citrus varieties. $V$ ATPase $H$ was annotated as two partial models and are both represented here separately as $V$-ATPase $H\left(5^{\prime}\right)$, denoting the 5-prime end of the gene, and V-ATPase $H\left(3^{\prime}\right)$, denoting the 3-prime end of the gene. Expression data were collected from the Citrus Greening 
Expression Network [9], with psyllid RNA-Seq data obtained from NCBI BioProject's PRJNA609978 and PRJNA448935, in addition to several published datasets [24-28]. Citrus hosts are abbreviated as Csin (Citrus sinensis), Cmed (Citrus medica), Cret (Citrus reticulata), and

Cmac

(Citrus

macrophylla).

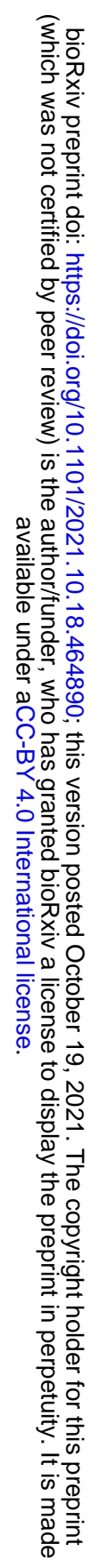


Grace et al. $\mid 23$

\section{Data Validation and Quality Control}

Genes encoding all 13 subunits required to build a single Vacuolar ATP synthase enzyme, as well as an accessory subunit S1 (Ac45) gene, were annotated in D. citri. There were no additional subunits found in D. citri, as reported in other metazoans [2]. Although insect VATPases are known to contain 13 subunits, there is variation in the gene copy number for individual subunits among different species (Tables 6-8). The $\mathrm{V}_{0}$ transmembrane domain subunits $V$-ATPase $a, b$, and $e$; the $\mathrm{V}_{1}$ catalytic domain subunits $V$-ATPase $A, C, D$, and $G$; and Ac45, all show variation in copy number among different species. The three Hemipterans analyzed (D. citri, A. pisum, and B. tabaci) maintain the same paralog number for all V-ATPase genes except for the A. pisum V-ATPase $D$ and $G$, as compared to the other orders (Table 7). This variation in copy number is interesting in contrast to the genes $V$-ATPase $c, d, B, E, F$, and $H$ that maintain only one gene copy across all Orders of Insecta found in Tables 6-8. V-ATPase subunits have been studied in plants, animals, fungi, and insects, and certain genes have been highlighted for their functional versatility in serving cell needs. For example, yeast and mammals have numerous copies and isoforms of the transmembrane proteolipid V-ATPase a with functions that support vacuoles, Golgi, neurons, osteoclasts, and epididymal cells [2]. D. citri, along with A. pisum and B. tabaci, has two copies of the V-ATPase a gene, whereas D. melanogaster has five copies (Table 6). In D. citri, a paralog of V-ATPase a was found, and they maintain differences in their amino acid sequences (Table 2, Table 6). Phylogenetic analysis of $\mathrm{V}_{0}$ subunit protein sequences supports that the duplication event occurred before the divergence of Hemimetabola and Holometabola (Figure 2). 
Grace et al. $\mid 24$

Table 6. Gene copy comparison of $\mathrm{V}_{0}$ transmembrane subunit V-ATPase genes in D. citri and orthologous insect genes.

\begin{tabular}{lccccc}
\hline \multirow{2}{*}{ Insect } & V-ATPase & V-ATPase & V-ATPase & V-ATPase & V-ATPase \\
& $a$ & $b$ & $c$ & $d$ & $e$ \\
\hline Diaphorina citri (Hemiptera) & 2 & 1 & 1 & 1 & 1 \\
Acyrthosiphon pisum (Hemiptera) & 2 & 1 & 1 & 1 & 1 \\
Bemisia tabaci (Hemiptera) & 2 & 1 & 1 & 1 & 1 \\
Tribolium castaneum (Coleoptera) & 2 & 2 & 1 & 1 & 2 \\
Drosophila melanogaster (Diptera) & 5 & 2 & 1 & 1 & 4 \\
Aedes aegypti (Diptera) & 3 & 1 & 1 & 1 & 2 \\
Apis mellifera (Hymenoptera) & 2 & 1 & 1 & 1 & 2 \\
\hline
\end{tabular}

Table 7. Gene copy comparison of $\mathrm{V}_{1}$ catalytic subunit V-ATPase genes in D. citri and orthologous insect genes.

\begin{tabular}{lcccccccc}
\hline & $V-$ & $V-$ & $V-$ & $V-$ & $V-$ & $V-$ & $V-$ & $V-$ \\
Insect & $A T P a$ & $A T P a$ & $A T P a$ & $A T P a$ & $A T P a$ & ATPa & ATPa & ATPa \\
& se A & se B & se C & se D & se E & se F & se G & se H \\
\hline Diaphorina citri (Hemiptera) & 1 & 1 & 1 & 1 & 1 & 1 & 1 & 1 \\
Acyrthosiphon pisum (Hemiptera) & 1 & 1 & 1 & 2 & 1 & 1 & 2 & 1 \\
Bemisia tabaci (Hemiptera) & 1 & 1 & 1 & 1 & 1 & 1 & 1 & 1 \\
Tribolium castaneum (Coleoptera) & 1 & 1 & 1 & 3 & 1 & 1 & 2 & 1 \\
Drosophila melanogaster (Diptera) & 2 & 1 & 1 & 3 & 1 & 1 & 1 & 1 \\
Aedes aegypti (Diptera) & 1 & 1 & 2 & 2 & 1 & 1 & 3 & 1 \\
Apis mellifera (Hymenoptera) & 1 & 1 & 1 & 2 & 1 & 1 & 1 & 1 \\
\hline
\end{tabular}

Table 8. Gene copy comparison of the Ac45 gene in D. citri and orthologous insect genes.

\begin{tabular}{lc}
\hline Insect & Ac45 \\
\hline Diaphorina citri (Hemiptera) & 1 \\
Acyrthosiphon pisum (Hemiptera) & 1 \\
Bemisia tabaci (Hemiptera) & 1 \\
Tribolium castaneum (Coleoptera) & 2 \\
Drosophila melanogaster (Diptera) & 2 \\
Aedes aegypti (Diptera) & 2 \\
Apis mellifera (Hymenoptera) & 1 \\
\hline
\end{tabular}


Grace et al. $\mid 25$

We were able to identify complete genes in genome v3.0 for all the subunits except $V$-ATPase $H$. Using genome-independent transcript sequences, we were able to determine that the 3' portion of the $V$-ATPase $H$ gene is located on chromosome 1 , but the 5 ' end of the gene is on one of the unplaced chromosomes that make up chromosome 0 (Table 1). Tables 2-4 show results of protein BLAST analysis comparing the same insects as found in Tables 6-8. Other than Ac45, all subunits share a relatively high identity, approximately 57-94\%, among individual pairwise alignments with each $D$. citri sequence (Tables 2-3). BLAST results of annotated gene models had high query coverage to orthologs supporting the completeness of the annotated gene models. In contrast, the sequence identities of Ac45, approximately 24-33\%, show the highest divergence when comparing $D$. citri to other insects (Table 4). For the $\mathrm{V}_{0}$, transmembrane domain, subunits in Table 2, proteolipid subunit c (V-ATPase $c)$ maintains some of the highest percentages of sequence identity, highlighting the importance of the protein function to form the c-ring that rotates and ultimately translocates protons across various membranes [2]. This is supported in Table 6, in which a single gene copy for $V$-ATPase $c$ is maintained across different orders of insects.

The Citrus Greening Expression Network (CGEN), found at citrusgreening.org, was used to compare transcript expression levels in various regions of $D$. citri which have either been exposed to or not exposed to CLas infection, the causative agent of citrus greening disease $[9,29,30]$. Figure 5 shows a heatmap comparing $\mathrm{V}_{0}$ subunit expression levels found under various conditions. V-ATPase $c$ is visually differentiated by its inflated expression levels as compared to other V-ATPase transmembrane subunit genes (Figure 5a). V-ATPase c expression also shows a 2.63-fold increase, from 647.9 to 1705.25 TPM, in the guts of adult psyllids fed on 
Grace et al. $\mid 26$

infected versus uninfected C. medica leaves (Figure 5b, Table 5). These expression levels, coupled with the fundamental cellular nature and relatively even occurrence of $V$-ATPases, suggest that V-ATPase genes are good candidates for RNAi. Silencing a $\mathrm{V}_{0}$ transcript should have inhibitory effects on the assembly of the V-ATPase enzyme. In particular, if infected psyllids increase in their demand for higher V-ATPase $c$ expression levels overall, knocking this transcript down will likely be detrimental for the insect. However, it cannot be determined at this time whether the elevated expression of $V$-ATPase $c$ relative to other subunits in infected psyllids is due to higher demand of these proteins in the cell and should therefore be studied further in future research.

Of the $\mathrm{V}_{1}$, catalytic domain, subunit genes, $V$-ATPase $A$ and $V$-ATPase $B$ maintain the highest percentages of sequence identity, consistent with the importance of their function in containing the ATP binding sites at the V-ATPase subunits A/B protein interface (Table 3) [31]. Apart from D. melanogaster, V-ATPase $A$ and $B$ also maintain single copies of these two genes across different orders of insects, supporting their conserved nature as compared to other genes of this enzyme (Table 7). When comparing expression of the $\mathrm{V}_{1}$ subunits, V-ATPase $A$ shows much higher expression compared to $V$-ATPase $B$ across each measured variable, with $V$-ATPase $G$ showing the highest expression in this group overall (Figure 6a). Unlike V-ATPase $c$, no significant differential expression was observed between the guts of insects reared on infected versus uninfected citrus trees (Figure 6b). However, V-ATPase B does show a reverse correlation, with a decrease in expression from 28.72 to 2.44 TPM in the whole body of $D$. citri nymphs raised on uninfected versus infected Citrus spp. (Table 5). A similar expression pattern can be seen throughout many of the V-ATPase catalytic genes and may infer an interaction 
Grace et al. $\mid 27$

between these genes and pathogen infection, therefore warranting further investigations (Table $5)$.

Figures 2, 3, and 4 depict phylogenetic analyses for the $\mathrm{V}_{0}$ transmembrane and $\mathrm{V}_{1}$ catalytic domains, and the Ac45 protein of V-ATPase, respectively. The individual V-ATPase subunits form clades, regardless of insect species. These clades also have the highest bootstrap values. This agrees with previous research that describes the enzyme as ancient and highly conserved. The evolution of V-ATPase has been analyzed for gene duplication and divergence from other ATP synthases, like F- and A-ATPase, which occur across the three domains of life [3]. Figures 2, 3, and 4 concur and suggest that the V-ATPase enzyme utilized in these insects existed in their common ancestor before they diverged into their respective species. The proteolipid subunit c and subunit d have the shortest branch lengths in Figure 2, consistent with Tables 2 and 6 which depict this to be of the most conserved subunits. Subunit c, which is required to form the critical c-ring rotor of V-ATPase [2], and subunit d, which may play a role as part of the central rotor of the V-ATPase [31], appear to have diverged the least when compared to the other transmembrane domain subunits and other insect species. In contrast, subunit e has diverged the most (Figure 3). This is consistent with the variable gene copy number observed across different orders of insects and the lower percentages of protein sequence identity seen in D. citri pairwise alignments (Tables 2,6). In addition, the function of subunit e is still unknown for the transmembrane domain subunits [5].

Figure 4 shows the evolutionary relatedness of the D. citri Ac45 protein. It is a relatively new protein critically associated with the assembly of a certain cell type V-ATPase and is still being studied [6]. For this select group of insect species, Ac45 groups and forms a clade with the other hemipteran protein sequences (Figure 4). Ac45 is a variable gene when comparing V-ATPase 
Grace et al. $\mid 28$

across the domains of life, a paralog variability that is also seen among different orders of insects

(Table 8) [6,32]. Ac45 has diverged most of all the V-ATPase subunits in D. citri compared to other insects. This divergence is seen in phylogenetic analysis, denoted with longer branch lengths (Figure 5), and is also supported in the values of the pairwise alignments, in which the protein shares very little sequence identity across the query lengths (Table 4). Perhaps it is experimentally beneficial that the Ac45 protein shows the least conservation with other insect orthologs. It may serve as a species-specific targeted approach to limiting the psyllid from vectoring the causative agent of citrus greening disease while leaving related species unharmed and their ecology intact. However, Ac45 shows a markedly depressed transcription level as compared to other subunits (Figure 6a, Table 5). This likely reflects the limits in resolution with current whole RNA isolation and sequencing methods but still indicates the relatively low total expression, nonetheless. The Ac45 protein has not been observed to exist in every cell type depending on the organism and so is not necessarily utilized by every V-ATPase in the psyllid [6]. Thus, the expression data agree with previously published research.

\section{Conclusion}

The V-ATPase is a fundamental enzyme that functions exclusively as ATP-dependent proton pumps in almost every eukaryotic cell. V-ATPase allows for the proper functioning of endosomes and the Golgi apparatus, and it generates a proton-motive force in organelles and across plasma membranes that is utilized as a driving force for secondary transport processes [1]. Identification of these enzymes in the hemipteran, D. citri, provides a novel insect lineage for studies of insect evolution and biology, and may also provide potential targets for $D$. citrispecific molecular mechanisms for the management of HLB in citrus production systems [33- 
Grace et al. $\mid 29$

35]. D. citri shows no deviation in the expected copy numbers of each of the V-ATPase genes (Tables 6-8). The data collected from D. citri reveals consistency among the genes previously characterized to be highly conserved, such as V-ATPase $c, d, A$, and $B$ (Tables 2-4) [3,31]. While expression data was not available for $V$-ATPase $d$, V-ATPase $c$ shows comparatively high expression levels overall and shows differential expression, 647.9 versus 1705.25 TPM, in the guts of adult psyllids fed on uninfected versus infected C. medica leaves (Figure 5, Table 5). Conversely, the Ac45 gene shows low expression throughout life stages and tissues compared to other V-ATPase genes, however, the highly divergent nature of this gene may serve as a speciesspecific targeted approach to psyllid control (Table 4, Figure 6).

In hemipterans, RNAi efficacy has been successfully demonstrated for psyllids, whitefly, and leafhoppers [33-40]; planthoppers [41,42]; bedbugs [43]; and others [44-48]. RNAi targeting specifically the V-ATPases in hemipteran insects have been reported for the corn planthopper, Peregrinus maidis (Ashmead) (Hemiptera: Delphacidae) [12]; the corn leafhopper, Dalbulus maidis (Hemiptera: Cicadellidae) [13]; the Brown planthopper, Nilaparvata lugens (Stål) (Hemiptera: Delphacidae) [41]; and the bedbug, Cimex lectularius L. (Hemiptera: Cimicidae) [43], resulting in increased mortality and reduced fecundity. Thus, the highly divergent nature of these gene sequences provides unique targets that may serve as species-specific targeting for RNAi approaches in the management of psyllid vectors and other hemipteran pests $[49,50]$.

\section{Reuse Potential}

The manually curated gene models generated through this $D$. citri community annotation project will be available as part of the Official Gene Set version 3. Analysis of this data, including 
Grace et al. $\mid 30$

BLAST and expression profiling, can be conducted using the citrusgreenin.org website and Citrus Greening Expression Network (CGEN). The improved annotations presented in this study will facilitate experimental design to investigate the potential of V-ATPases as gene targets for therapies to control D. citri. Research considering differential expression patterns on V-ATPase transcripts in psyllids fed on CLas infected plants should be conducted. Additional studies are also required to confirm the role of the Ac45 protein, as its divergent nature may provide novel and species-specific gene targets, potentially through the use of RNAi, to control psyllid populations and reduce the effects of pathogens such as CLas.

\section{Declarations}

\section{List of Abbreviations}

ACP: Asian citrus psyllid; V-ATPase: Vacuolar (H+)-ATP synthase; $\mathrm{V}_{0}$ : V-ATPase noncatalytic transmembrane domain; $\mathrm{V}_{1}$ : V-ATPase catalytic cytoplasmic domain; ATP: Adenosine triphosphate; ADP: Adenosine diphosphate; RNAi: RNA interference; HLB: Huanglongbing; CLas: Candidatus Liberibacter asiaticus; HGNC: HUGO Gene Nomenclature Committee; NCBI: National Center for Biotechnology Information; MCOT: Maker, Cufflinks, Oasis, Trinity; BLASTp: protein BLAST; RNA-seq: RNA sequencing; DNA-seq: DNA sequencing; Iso-seq: Isoform sequencing; OGS: Official Gene Set; ChrXX: Chromosome number location of OGS; QC: Query coverage; CGEN: Citrus Greening Expression Network; TPM: Transcripts per million; Csin: Citrus sinensis; Cmed: Citrus medica; Cret: Citrus reticulata; Cmac: Citrus macrophylla 


\section{Ethical Approval}

Not applicable.

\section{Consent for publication}

Not applicable.

\section{Competing Interests}

The authors declare that they have no competing interests.

\section{Funding}

This work was supported by USDA-NIFA grants 2015-70016-23028, HSI 2020-38422-

32252 and 2020-70029-33199.

\section{Authors' contributions}

WBH, SJB, TD, and LAM conceptualized the study; TD, SS, TDS, and SJB supervised the study; SJB, TD, SS, and LAM contributed to project administration; RG conducted investigation; PH, MF-G, and SS contributed to software development; PH, MF-G, SS, TDS, and JB developed methodology; SJB, TD, WBH, and LAM acquired funding; RG and CM prepared and wrote the original draft; TD, SJB, SS, TDS, WT, WBH and JB reviewed and edited the draft. 
Grace et al. $\mid 32$

\section{Acknowledgments}

We would like to thank Helen Wiersma-Koch (Indian River State College) for her assistance.

\section{References}

1. Nelson N, Perzov N, Cohen A, Hagai K, Padler V, Nelson H, The cellular biology of protonmotive force generation by V-ATPases. J Exp Biol. 2000; 203: 89-95.

2. Maxson ME, Grinstein S, The vacuolar-type H+-ATPase at a glance - more than a proton pump. J Cell Sci., 2014; 127: 4987-4993.

3. Hilario E, Gogarten JP, The prokaryote-to-eukaryote transition reflected in the evolution of the V/F/A-ATPase catalytic and proteolipid subunits. J Mol Evo., 1998; 46: 703-715.

4. Badillo-Vargas IE, Rotenberg D, Schneweis BA, Whitfield AE, RNA interference tools for the western flower thrips, Frankliniella occidentalis. J Insect Physiol., 2015; 76: 36-46.

5. Beyenbach KW, Wieczorek H, The V-type H+ ATPase: molecular structure and function, physiological roles and regulation. J Experim Bio., 2006; 209: 577-589.

6. Jansen EJ, van Bakel NH, Coenen AJ, van Dooren SH, van Lith HA, Martens GJ, An isoform of the vacuolar (H(+))-ATPase accessory subunit Ac45. Cell Mol Life Sci., 2010; 67: 629.

7. Dow JA, Davies SA, Guo Y, Graham S, Finbow ME, Kaiser K, Molecular genetic analysis of V-ATPase function in Drosophila melanogaster. J Exp Biol., 1997; 200: 237-245.

8. National Center for Biotechnology Information (NCBI). Ref-Seq Non-redundant Protein Database. Bethesda, MD: NCBI. https://www.ncbi.nlm.nih.gov/refseq/about/nonredundantproteins/. Accessed 2019. 
9. Flores-Gonzalez M, Hosmani PS, Fernandez-Pozo N, Mann M, Humann JL, Main D, et al. Citrusgreening.org: An open access and integrated systems biology portal for the Huanglongbing (HLB) disease complex. bioRxiv, 2019; https://doi.org/10.1101/868364.

10. Madeira F, Park YM, Lee J, Buso N, Gur T, Madhusoodanan N, et al. The EMBL-EBI search and sequence analysis tools APIs in 2019. Nucleic Acids Research, 2019; 47(W1): W636-W641.

11. Shippy T, Miller S, Massimino C, Vosberg C, Hosmani PS, Flores-Gonzalez M, et al. Annotating genes in Diaphorina citri genome version 3. protocols io. 2020; https://dx.doi.org/10.17504/protocols.io.bniimcce.

12. Yao J, Rotenberg D, Afsharifar A, Barandoc-Alviar K, Whitfield AE, Development of RNAi methods for Peregrinus maidis, the corn planthopper. PLoS One, 2013; 8(8): e70243.

13. Jones T-KL, Bernal JS, Medina RF, Assessing the Functionality of RNA Interference (RNAi) in the Phloem-feeding Maize pest Dalbulus maidis. bioRxiv, 2021; http://dx.doi.org/10.1101/2021.09.29.462424.

14. International Aphid Genomics Consortium. Genome sequence of the pea aphid Acyrthosiphon pisum. PLoS Biol., 2010; 8: e1000313.

15. Li Y, Park H, Smith TE, Moran NA, Gene Family Evolution in the Pea Aphid Based on Chromosome-Level Genome Assembly. Mol Biol Evol., 2019; 36: 2143-2156.

16. Chen W, Hasegawa DK, Kaur N, Kliot A, Pinheiro PV, Luan J, et al. The draft genome of whitefly Bemisia tabaci MEAM1, a global crop pest, provides novel insights into virus transmission, host adaptation, and insecticide resistance. BMC Biol., 2016; 14: 110 
17. Matthews BJ, Dudchenko O, Kingan SB, Koren S, Antoshechkin I, Crawford JE, et al. Improved reference genome of Aedes aegypti informs arbovirus vector control. Nature, 2018; 563: 501-507.

18. Elsik CG, Worley KC, Bennett AK, Beye M, Camara F, Childers CP, et al. Finding the missing honey bee genes: lessons learned from a genome upgrade. BMC Genomics, 2014; $15: 86$

19. Richards S, Gibbs RA, Weinstock GM, Brown SJ, Denell R, et al. Tribolium Genome Sequencing Consortium. The genome of the model beetle and pest Tribolium castaneum. Nature, 2008; 452: 949-955.

20. Thurmond J, Goodman JL, Strelets VB, Attrill H, Gramates LS, Marygold SJ, et al. FlyBase 2.0: the next generation. Nucleic Acids Res., 2019; 47: D759-D765.

21. Kumar S, Stecher G, Tamura K, MEGA7: Molecular Evolutionary Genetics Analysis Version 7.0 for Bigger Datasets. Mol Biol Evol., 2016; 33: 1870-1874.

22. $\mathrm{R}$ Core Team. R: A language and environment for statistical computing. Vienna: $\mathrm{R}$ Foundation for Statistical Computing 2020.

23. Kolde R, pheatmap: Pretty Heatmaps (Version 1.0.12). 2020; https://cran.rproject.org/package=pheatmap.

24. Reese J, Christenson MK, Leng N, Saha S, Cantarel B, Lindeberg M, et al. Characterization of the Asian Citrus Psyllid Transcriptome. J Genomics, 2014; 2: 54-58.

25. Wu Z, Zhang H, Bin S, Chen L, Han Q, Lin J, Antennal and abdominal transcriptomes reveal chemosensory genes in the Asian citrus psyllid, Diaphorina citri. PLoS One, 2016; 11: $\mathrm{e} 0159372$. 
26. Kruse A, Fattah-Hosseini S, Saha S, Johnson R, Warwick E, Sturgeon K, et al. Combining 'omics and microscopy to visualize interactions between the Asian citrus psyllid vector and the Huanglongbing pathogen Candidatus Liberibacter asiaticus in the insect gut. PLoS One, 2017; 12: e0179531.

27. Vyas M, Fisher TW, He R, Nelson W, Yin G, Cicero JM, et al. Asian citrus psyllid expression profiles suggest Candidatus Liberibacter asiaticus-mediated alteration of adult nutrition and metabolism, and of nymphal development and immunity. PLoS One, 2015; 10: e0130328.

28. Yu H-Z, Li N-Y, Zeng X-D, Song J-C, Yu X-D, Su H-N, et al. Transcriptome analyses of Diaphorina citri midgut responses to Candidatus Liberibacter asiaticus infection. Insects, 2020; 11: 171.

29. Hosmani PS, Flores-Gonzalez M, Shippy T, Vosburg C, Massimino C, Tank W, et al. Chromosomal length reference assembly for Diaphorina citri using single-molecule sequencing and Hi-C proximity ligation with manually curated genes in developmental, structural and immune pathways. bioRxiv, 2019; https://doi.org/10.1101/869685.

30. Duan Y, Zhou L, Hall DG, Li W, Doddapaneni H, Lin H, et al. Complete genome sequence of citrus huanglongbing bacterium, "Candidatus Liberibacter asiaticus" obtained through metagenomics. Mol Plant Microbe Interact, 2009; 22(8): 1011-1020.

31. Toei M, Saum R, Forgac M, Regulation and isoform function of the V-ATPases. Biochemistry, 2010; 49: 4715-4723.

32. Forgac M, Vacuolar ATPases: rotary proton pumps in physiology and pathophysiology. Nat Rev Mol Cell Biol., 2007; 8: 917-929. 
33. Hunter WB, Clarke S-KV, Mojica AFS, Paris TM, Miles G, Metz JL, et al. Advances in RNA suppression of the Asian citrus psyllid vector and bacteria (huanglongbing pathosystem). Asian Citrus Psyllid: Biology, Ecology and Management of the Huanglongbing Vector Wallingford: CABI. 2020; 258-84.

34. Hunter WB, Cooper WR, Sandoval-Mojica AF, McCollum G, Aishwarya V, Pelz-Stelinski KS, Improving Suppression of Hemipteran Vectors and Bacterial Pathogens of Citrus and Solanaceous Plants: Advances in Antisense Oligonucleotides (FANA). Frontiers in Agronomy. 2021; 3: 48.

35. Hunter WB, Wintermantel WM, Optimizing Efficient RNAi-Mediated Control of Hemipteran Pests (Psyllids, Leafhoppers, Whitefly): Modified Pyrimidines in dsRNA Triggers. Plants, 2021; 10(9): 1782.

36. Andrade EC, Hunter WB, RNAi feeding bioassay: development of a non-transgenic approach to control Asian citrus psyllid and other hemipterans. Entomol Exp Appl., 2017; 162: 389-396.

37. Li H, Guan R, Guo H, Miao X, New insights into an RNAi approach for plant defence against piercing-sucking and stem-borer insect pests. Plant Cell Environ., 2015; 38(11): $2277-2285$.

38. Liu X, Zou Z, Zhang C, Liu X, Wang J, Xin T, et al. Knockdown of the Trehalose-6Phosphate Synthase Gene Using RNA Interference Inhibits Synthesis of Trehalose and Increases Lethality Rate in Asian Citrus Psyllid, Diaphorina citri (Hemiptera: Psyllidae). Insects, 2020; 11(9): 605. 
39. Luo Y, Chen Q, Luan J, Chung SH, Van Eck J, Turgeon R, et al. Towards an understanding of the molecular basis of effective RNAi against a global insect pest, the whitefly Bemisia tabaci. Insect Biochem Mol Biol., 2017; 88: 21-29.

40. Yu X, Killiny N, RNA interference of two glutathione S-transferase genes, Diaphorina citri DcGSTe2 and DcGSTd1, increases the susceptibility of Asian citrus psyllid (Hemiptera: Liviidae) to the pesticides fenpropathrin and thiamethoxam. Pest Manag Sci., 2018; 74(3): $638-647$.

41. Li J, Chen Q, Lin Y, Jiang T, Wu G, Hua H, RNA interference in Nilaparvata lugens (Homoptera: Delphacidae) based on dsRNA ingestion. Pest Manag Sci., 2011; 67(7): 852859.

42. Li J, Wang X-P, Wang M-Q, Ma W-H, Hua H-X, Advances in the use of the RNA interference technique in Hemiptera. Insect Sci., 2013; 20(1): 31-39.

43. Basnet S, Kamble ST, RNAi-Mediated Knockdown of vATPase Subunits Affects Survival and Reproduction of Bed Bugs (Hemiptera: Cimicidae). J Med Entomol., 2018; 55(3): 540_ 546.

44. Cagliari D, Dias NP, Galdeano DM, Dos Santos EÁ, Smagghe G, Zotti MJ, Management of Pest Insects and Plant Diseases by Non-Transformative RNAi. Front Plant Sci., 2019; 10: 1319.

45. Christiaens O, Smagghe G, The challenge of RNAi-mediated control of hemipterans. Curr Opin Insect Sci., 2014; 6: 15-21.

46. Eakteiman G, Moses-Koch R, Moshitzky P, Mestre-Rincon N, Vassão DG, Luck K, et al. Targeting detoxification genes by phloem-mediated RNAi: A new approach for controlling phloem-feeding insect pests. Insect Biochem Mol Biol., 2018; 100: 10-21. 
Grace et al. $\mid 38$

47. Fletcher SJ, Reeves PT, Hoang BT, Mitter N, A Perspective on RNAi-Based Biopesticides. Front Plant Sci., 2020; 11: 51.

48. Jain RG, Robinson KE, Fletcher SJ, Mitter N, RNAi-Based Functional Genomics in Hemiptera. Insects, 2020; 11(9): 557.

49. Whyard S, Singh AD, Wong S, Ingested double-stranded RNAs can act as species-specific insecticides. Insect Biochem Mol Biol., 2009; 39(11): 824-832.

50. Christiaens O, Whyard S, Vélez AM, Smagghe G, Double-Stranded RNA Technology to Control Insect Pests: Current Status and Challenges. Front Plant Sci., 2020; 11: 451. 